\title{
Exposição ocupacional dos profissionais de enfermagem de uma unidade de terapia intensiva a material biológico ${ }^{1}$
}

\section{Nursing team occupational exposure at an intensive care unit a biological material}

\author{
Exposición ocupacional de profesionales de enfermería de una \\ unidad de terapia intensiva a material biológico
}

\author{
Aline Maria Bonini', Camila de Paula Zeviani", Luiza Tayar Facchin"', \\ Elucir Girlv, Silvia Rita Marin da Silva Canini ${ }^{\vee}$
}

\footnotetext{
1 Artigo extraído da monografia de conclusão do curso de Especialização em Controle de Infecção em Serviços de Saúde da Escola de Enfermagem de Ribeirão Preto da Universidade de São Paulo (EERP/USP).

' Enfermeira do Hospital das Clínicas da Faculdade de Medicina de Ribeirão Preto da USP. E-mail: alinebonini@bol.com.br.

"Enfermeira da Irmandade de Misericórdia de Sertãozinho. E-mail: kmilaz@zipmail.com.br.

II' Enfermeira. Mestranda do Programa de Enfermagem Fundamental da EERP/USP. E-mail: luiza@eerp.usp.br.

IV Enfermira. Professora Titular da EERP/USP. E-mail: egir@eerp.usp.br

$\checkmark$ Enfermeira. Professora Doutora do Departamento de Enfermagem Geral e Especializada da Escola de Enfermagem da USP. E-mail: canini@eerp.usp.br.
}

\section{RESUMO}

Este estudo descritivo exploratório teve como objetivo caracterizar os profissionais de enfermagem de um centro de terapia intensiva que sofreram acidentes ocupacionais com material biológico e os acidentes. Os dados foram coletados no período de maio a junho de 2007, por meio de entrevista individual utilizando-se um roteiro semi-estruturado. Foram entrevistados 37 profissionais sendo que $25(67,56 \%)$ referiram ter sofrido acidente com material biológico, e a maioria era técnico de enfermagem, do sexo feminino, entre 21 a 30 anos e com experiência na enfermagem menor que 05 anos. Em relação à capacitação sobre prevenção deste tipo de exposição $28(75,7 \%)$ afirmaram ter participado e apenas 14 (56\%) sujeitos referiram que utilizavam equipamento de proteção individual no momento do acidente. A maioria das exposições 18 (72\%) foi percutânea e o sangue foi o fluido mais envolvido 17(68\%). As causas mais frequentemente atribuídas à ocorrência dos acidentes foram: falta de atenção e pressa. Apesar dos profissionais alegarem ter recebido capacitação durante sua formação e atuação profissional, observa-se que tiveram baixa adesão ao uso de equipamentos de proteção individual, evidenciando a necessidade de rever o processo de trabalho e as estratégias utilizadas nos programas educativos.

Descritores: Enfermagem; Unidade de terapia intensiva; Acidentes ocupacionais.

\section{ABSTRACT}

This descriptive and exploratory study aimed to characterize nursing professionals from an intensive care unit who were victims of occupational accidents involving biological material, as well as the accidents. Data were collected between May and June of 2007, by means of individual interviews with a semistructured script. Thirty-seven professionals were interviewed, 25 $(67.56 \%)$ of whom mentioned having been victims of an accident with biological material, and most of them were female nursing technicians, between 21 and 30 years old, who had less than 05 years of nursing experience. As to capacitation about how to prevent this type of exposure, $28(75.7 \%)$ affirmed that they had received it, and only 14 (56\%) subjects indicated that they were using individual protection equipment at the time of the accident. Most cases of exposure, 18 ( $72 \%$ ) were percutaneous and blood was the most involved fluid, in $17(68 \%)$ cases. The most frequently causes attributed to the occurrence of accidents were: lack of attention and hurry. Although the professionals alleged they had received capacitation during their education and professional activity, adherence levels to the use of individual protection equipment were low, evidencing the need to reconsider the work process and the strategies used in education programs.

Descriptors: Nursing; Intensive care unit; Occupational accidents.

\section{RESUMEN}

La finalidad de este estudio descriptivo exploratorio fue caracterizar los profesionales de enfermería de un centro de terapia intensiva que sufrieron accidentes de trabajo con material biológico y los accidentes. Los datos fueron recolectados en el período de mayo a junio de 2007, mediante entrevista individual utilizándose un guión semiestructurado. Se les entrevistaron a 37 profesionales, siendo que $25(67,56 \%)$ refirieron haber sufrido accidente con material biológico, y la mayoría era técnico de enfermería, del género femenino, entre 21 y 30 años y con experiencia en la enfermería menor de 05 años. Respecto a la capacitación sobre prevención de este tipo de exposición, 28 (75,7\%) afirmaron que lo recibieron y sólo 14 (56\%) sujetos indicaron que utilizaban equipo de protección individual en el momento del accidente. La mayoría de las exposiciones 18 (72\%) fue percutánea y la sangre fue el fluido más involucrado 17 (68\%). Las causas más frecuentemente atribuidas a la ocurrencia de los accidentes fueron: falta de atención y prisa. Aunque los profesionales alegaron haber recibido capacitación durante su formación y actuación profesional, se observa que tuvieron baja adhesión al uso de equipos de protección individual, evidenciando la necesidad de rever el proceso de trabajo y las estrategias utilizadas en los programas educativos. Descriptores: Enfermería; Unidad de terapia intensiva; Accidentes de trabajo. 


\section{NTRODUÇÃO}

Nas últimas duas décadas, os acidentes ocupacionais envolvendo material biológico e trabalhadores da área da saúde vêm sendo foco crescente de pesquisas, uma vez que este tipo de exposição pode levá-los a contrair infecções, e consequentemente, sérios agravos à sua saúde.

A aquisição dos vírus das hepatites B e C (HBV e HCV) e do Vírus da Imunodeficiência Humana (HIV) por trabalhadores da área da saúde já está comprovada.

Segundo dados disponíveis até setembro de 1997, foram registrados 264 casos de aquisição do HIV com a seguinte distribuição: $55,4 \%$ dos casos nos Estados Unidos, $11,7 \%$ na França, 5,3\% na Itália, 5,3\% na Espanha, 4,4\% na Austrália, 4,2\% na Inglaterra, 3,2\% na África do Sul e o restante dos casos, em menor percentual, em outros países ${ }^{(1)}$.

Estudo realizado nos Estados Unidos ${ }^{(2)}$ demonstra que já existem 57 casos documentados de aquisição ocupacional do HIV, sendo que 24 (42\%) casos ocorreram com enfermeiros. No Brasil, o primeiro caso de AIDS adquirido de maneira ocupacional só foi reconhecido pelo Ministério do Trabalho, em 1999, acometendo uma auxiliar de enfermagem que auxiliava uma colega no procedimento de punção venosa, até o momento existem quatro casos confirmados de transmissão ocupacional, todos com trabalhadores de enfermagem $^{(3)}$.

O sangue e todos os fluidos corporais com sangue visível oferecem risco de transmitir diferentes patógenos, sendo os vírus das hepatites $\mathrm{B}$ e $\mathrm{C}$ e o HIV, os de maior relevância epidemiológica.

O risco para a aquisição de patógenos veiculados é variável e depende do tipo de acidente, da gravidade e tamanho da lesão, presença e volume do sangue no ato do acidente, e também, as condições clínicas do paciente-fonte e o uso correto da profilaxia após a exposição. O risco de infecção por HIV pós-exposição ocupacional percutânea é de aproximadamente $0,3 \%$, já pelo HCV é de $1,8 \%$, podendo variar de 0 a $7 \%$ e do HBV após acidente percutâneo envolvendo paciente fonte HbeAg positivo está estimado entre 6 e 30\%, podendo atingir até $40 \%$, quando nenhuma medida profilática é $\operatorname{adotada}^{(4)}$.

Apesar do risco de transmissão desses vírus ser conhecido há alguns anos, inclusive com recomendações de medidas preventivas foi somente com o advento da AIDS que maior ênfase passou a ser dada a essas medidas.

As precauções-padrão ${ }^{(5-6)}$ se aplicam ao cuidado de todos os pacientes, independentemente do seu diagnóstico, recomendam o uso de luvas, aventais, máscaras e protetores oculares, sempre que o contato com fluidos corporais for previsto. E reforça também a higienização das mãos antes e após o contato com pacientes e fluidos corpóreos, bem como antes e após o uso de luvas. A adesão às precauçõespadrão é considerada uma das mais importantes medidas profiláticas para evitar a exposição.

Em relação às medidas preventivas préexposição, a imunização contra a hepatite $B$ é considerada como uma das medidas preventivas mais importantes para a prevenção do HBV, com eficácia de 90 a 95\%. E quanto às medidas pós-exposição ao HBV, no caso do profissional não vacinado é recomendada a imunoglobulina hiperimune contra hepatite B (IGHAHB) que confere $75 \%$ de efetividade na prevenção dessa infecção(4).

Para exposições envolvendo paciente-fonte positivo para o HIV existe a recomendação de quimioprofilaxia com anti-retrovirais ${ }^{(7)}$. E no caso do $\mathrm{HCV}$, as medidas se restringem à utilização das precauções-padrão(5-6) , uma vez que até o momento não existe outra medida eficaz para reduzir o risco de sua transmissão.

Estudo realizado sobre acidentes ocupacionais com material biológico entre a equipe de enfermagem $^{(8)}$ de um hospital escola do interior paulista, identificou que a maioria dos acidentes com material biológico ocorreu por via percutânea, os mais atingidos foram os trabalhadores de enfermagem e em muitas situações as precauçõespadrão não foram obedecidas.

Os indivíduos que trabalham em Unidades de Terapia Intensiva são mais acometidos por acidentes envolvendo materiais biológicos potencialmente contaminados, não só pelo grande número de procedimentos, mas também devido ao ritmo mais intenso de trabalho ${ }^{(9)}$.

Destaca-se que no centro de terapia intensiva estão os pacientes mais críticos o que por sua vez demanda da equipe de saúde e principalmente da equipe de enfermagem um ritmo de trabalho intenso, inúmeras intervenções com manuseio constante de material biológico e perfurocortante o que consequentemente aumenta o risco de exposição. Com o intuito de contribuir para o direcionamento de estratégias preventivas específicas para estes trabalhadores julgou-se oportuna a realização do presente estudo que teve como objetivos caracterizar o perfil dos profissionais de enfermagem que sofreram acidentes ocupacionais com material biológico e os respectivos acidentes.

\section{METODOLOGIA}

Trata-se de um estudo descritivo exploratório de caráter prospectivo, realizado num hospital filantrópico integrado ao Sistema Único de Saúde (SUS) de uma cidade do interior de São Paulo, que dispõe de 144 leitos para internação, e de uma Unidade de Terapia Intensiva (UTI) com nove leitos. 
A equipe de enfermagem da UTI é composta por 21 auxiliares e técnicos de enfermagem e 16 enfermeiros, sendo que os enfermeiros também cobrem outras áreas do hospital.

Nesta instituição existe um protocolo de atendimento para os profissionais que sofrem acidente ocupacional envolvendo material biológico e as fichas de notificação destes acidentes são encaminhadas ao setor de Recursos Humanos que juntamente ao técnico de segurança do trabalho preenchem a Comunicação de Acidente de Trabalho (CAT) e as enviam ao Instituto Nacional do Seguro Social (INSS).

A população do estudo foi composta por todos os trabalhadores de enfermagem da UTI que estavam em exercício ativo nesse hospital, no período do estudo e concordaram em participar mediante assinatura do termo de consentimento livre e esclarecido.

Os dados foram coletados no período de 01 de maio a 30 de junho de 2007, por meio de entrevista individual norteada por um instrumento de coleta de dados contendo questões abertas e fechadas. O banco de dados foi estruturado e analisado por meio do programa para microcomputador Statistical
Package Social Science (SPSS) versão 10.0 for Windows (SPSS, 1999), utilizando-se estatística descritiva.

Após o consentimento da Chefia de Enfermagem e da Diretoria Clínica do referido hospital o projeto foi submetido à apreciação do Comitê de Ética e Pesquisa da Escola de Enfermagem de Ribeirão Preto da Universidade de São Paulo, sendo aprovado (protocolo 0781/2007).

\section{RESULTADOS E DI SCUSSÃO}

Os dados referentes à caracterização dos trabalhadores de enfermagem que sofreram acidentes com material biológico estão descritos na Tabela 1. Do total de 37 sujeitos entrevistados, 25 sofreram acidentes com material biológico $(67,6 \%)$, sendo que 08 (32\%) eram enfermeiros, 16 (64\%) técnicos de enfermagem e 01 (4\%) auxiliar de enfermagem, sendo que a maioria era do sexo feminino 19 (76\%), com idade de 21 a 30 anos $(60 \%)$ e tempo de experiência profissional na enfermagem 16 (64\%) tinham $\leq 5$ anos. Em relação à jornada de trabalho, 13 (52\%) sujeitos trabalhavam $\geq 51$ horas/semana.

Tabela 1: Caracterização dos trabalhadores de enfermagem ( $N=25)$ que sofreram acidentes com material biológico $(\mathrm{N}=25)$ de um Centro de Terapia Intensiva, segundo as variáveis do estudo. Sertãozinho, 2007.

\begin{tabular}{|c|c|c|}
\hline Variáveis & No & $\%$ \\
\hline \multicolumn{3}{|l|}{ Categoria profissional } \\
\hline Enfermeiro & 08 & 32,0 \\
\hline Técnico de enfermagem & 16 & 64,0 \\
\hline Auxiliar de enfermagem & 01 & 4,0 \\
\hline \multicolumn{3}{|l|}{ Sexo } \\
\hline Feminino & 19 & 76,0 \\
\hline Masculino & 06 & 24,0 \\
\hline \multicolumn{3}{|l|}{ I dade (anos) } \\
\hline$<21$ & 02 & 8,0 \\
\hline $21|-| 30$ & 15 & 60,0 \\
\hline $31|-| 40$ & 03 & 12,0 \\
\hline$\geq 41$ & 05 & 20,0 \\
\hline \multicolumn{3}{|c|}{ Tempo de Experiência na Enfermagem (anos) } \\
\hline$\leq 05$ & 16 & 64,0 \\
\hline $06|-| 10$ & 06 & 24,0 \\
\hline$\geq 11$ & 03 & 12,0 \\
\hline \multicolumn{3}{|c|}{ J ornada de trabalho (horas/ semana) } \\
\hline$\leq 40$ & 10 & 40,0 \\
\hline $41|-| 50$ & 08 & 8,0 \\
\hline$\geq 51$ & 13 & 52,0 \\
\hline \multicolumn{3}{|l|}{ Turno de trabalho } \\
\hline Diurno fixo & 10 & 40,0 \\
\hline Noturno fixo & 09 & 36,0 \\
\hline Rodízio & 06 & 24,0 \\
\hline \multicolumn{3}{|l|}{ Capacitação } \\
\hline Sim & 20 & 80,0 \\
\hline Não & 05 & 20,0 \\
\hline
\end{tabular}


Estudo de corte transversal ${ }^{(10)}$, realizado em um hospital de Hong Kong, avaliou o conhecimento e a adesão à precauções universais de 306 enfermeiros, e revelou que tanto os níveis de conhecimento quanto à adesão às precauções universais foram baixos e que enfermeiros com maior tempo de experiência profissional obtiveram maiores escores de adesão que àqueles com menos de 05 anos de experiência.

Ainda em relação ao tempo de experiência profissional na enfermagem, estudo sobre o clima organizacional e pessoal, e, também os equipamentos de proteção individual ${ }^{(11)}$ apontou que para cada ano de prática na enfermagem, o risco de acidente decrescia progressivamente e que enfermeiros com menos de 05 anos de experiência profissional tiveram 1,48 mais chance de se acidentarem.

Observa-se que quanto ao turno de trabalho, 10 $(40 \%)$ trabalham no diurno fixo, $09(36 \%)$ noturno fixo e 06 ( $24 \%$ ) em rodízio de turnos.

Estudo descritivo ${ }^{(12)}$ sobre acidentes de trabalho com material perfurocortante entre a equipe de enfermagem de um hospital universitário, encontrou maior freqüência de acidentes nesse mesmo turno, atribuindo-os ao ritmo de trabalho mais intenso no período diurno, em relação ao noturno.

A maioria dos sujeitos $20(80 \%)$ relatou ter participado de cursos de capacitação relacionados à prevenção de acidente ocupacional com material biológico há menos de um ano, mais que uma vez e que o mesmo ocorreu na própria instituição onde trabalham. Estudo de caso-controle ${ }^{(13)}$ evidenciou que o treinamento em prevenção de acidentes foi um fator de risco independente para a ocorrência de acidentes com material perfurocortante e que trabalhar em centro de terapia intensiva aumentou o risco de acidentes em cerca de três vezes $(\mathrm{OR}=3,48$; IC95\%:1,37-8,85). A variável sobre capacitação do pessoal sobre prevenção de acidentes precisa ser mais bem investigada, pois em relação à capacitação pontual, pesquisa ${ }^{(14)}$ mostrou que a taxa de adesão às precauções-padrão aumentou durante a intervenção, porém não foi suficiente para manter os níveis de adesão, os quais declinaram no período pós-intervenção, sendo o efeito deste tipo de capacitação considerado temporário. Esse fato mostra a necessidade de programas de educação permanente ao invés de capacitações e cursos esporádicos.

Segundo situação vacinal contra Hepatite B, 25 $(100 \%)$ relataram ter recebido as três doses da vacina, no hospital do estudo, existe um monitoramento sobre a carteira de vacinação dos trabalhadores da área da saúde, instituído desde 2004. Após diversas campanhas sucessivas sobre a importância da vacinação como medida preventiva contra a hepatite $B$, houve aumento progressivo da adesão dos profissionais a esta medida, alcançando o índice de $100 \%$.

Observa-se, na Tabela 2, que a maioria das exposições 18 (72\%) foi percutânea, dados semelhantes têm sido apontado na literatura ${ }^{(8,15)}$, e que a exposição de mucosas é o segundo tipo mais freqüente, seguido por exposição de pele não íntegra e um estudo ${ }^{(15)}$ encontrou acidentes ocasionados por mordida de pacientes.

Tabela 2: Caracterização dos acidentes com material biológico $(N=25)$ sofrido por trabalhadores de enfermagem de um Centro de Terapia Intensiva, segundo as variáveis do estudo. Sertãozinho, 2007.

\begin{tabular}{lcc}
\hline \multicolumn{1}{c}{ Variáveis } & $\mathbf{N} 0$ & $\%$ \\
\hline Tipo de exposição & 18 & 72,0 \\
Percutânea & 05 & 20,0 \\
Cutânea & 02 & 8,0 \\
Mucosa & & \\
Número de exposições & 11 & 44,0 \\
Uma & 05 & 20,0 \\
Duas & 06 & 24,0 \\
Várias & 03 & 12,0 \\
Não soube informar & & \\
Fluido corporal envolvido & 17 & 68,0 \\
Sangue & 05 & 20,0 \\
Sangue e outro fluido & 01 & 4,0 \\
Secreção de vias aéreas & 01 & 4,0 \\
Secreção gástrica & 01 & 4,0 \\
Não soube informar & & \\
Uso de Equipamento de Proteção Individual & 14 & 56,0 \\
Sim & 11 & 44,0 \\
Não & & \\
\hline
\end{tabular}


Em relação ao número de exposições, 11 sujeitos sofreram uma única exposição, 05 sujeitos 02 exposições e 06 sujeitos relataram ter sofrido várias exposições.

O fluido corporal mais envolvido foi o sangue em $17(68 \%)$ exposições e $11(44 \%)$ dos trabalhadores não usavam equipamento de proteção individual na ocasião do acidente.

O motivo mais frequentemente atribuído ao não uso do equipamento de proteção individual foi a situação de urgência por $05(45,4 \%)$ sujeitos, seguida por falta de tempo e devido ao procedimento não exigir o uso (Tabela 3 ).

Tabela 3: Distribuição do número e porcentagem dos trabalhadores de enfermagem de um Centro de Terapia Intensiva que sofreram exposição ocupacional a material biológico que não utilizavam o equipamento de proteção individual ( $\mathrm{N}=11$ ), segundo o motivo atribuído. Sertãozinho, 2007.

\begin{tabular}{lccc}
\hline & Motivo & No & \% \\
\hline Situação de Urgência & & \\
Falta de tempo & 05 & 45,4 \\
Procedimento não exigia & 03 & 27,3 \\
Situação de Urgência & 03 & 27,3 \\
\hline Total & $\mathbf{1 1}$ & $\mathbf{1 0 0 , 0}$ \\
\hline
\end{tabular}

Observa-se que 11 (44\%) sujeitos referiram não estar usando equipamento de proteção individual no momento do acidente. Outra pesquisa ${ }^{(16)}$ realizada numa unidade de terapia intensiva, encontrou que $60,0 \%$ dos trabalhadores não faziam uso de equipamento de proteção individual no momento do acidente. Dados semelhantes foram encontrados num estudo $^{(17)}$ com profissionais da equipe de resgate do corpo de bombeiros de Goiás, que atribuíram como fatores dificultadores para a utilização do EPI, a sobrecarga de trabalho $(72,7 \%)$, falta de material $(36,3 \%)$, falta de incentivo $(29,5 \%)$, situações de emergência e falta de tempo $(20,4 \%)$.

Segundo a NR 32/2005 é dever da empresa fornecer o EPI em quantidade e qualidade. E cabe ao trabalhador utilizar o EPI apenas com a finalidade a que se destina, responsabilizar-se por sua guarda e conservação, além de comunicar ao empregador qualquer dano ou alteração que o torne impróprio para o uso(18). A análise da utilização de EPI é complexa, pois existem fatores relacionados ao empregador, ao empregado e ao próprio EPI.

Quanto as causas atribuídas, pelos trabalhadores, para a ocorrência de exposição ocupacional a material biológico, 09 (33,4\%) referiram falta de atenção, $04(14,8 \%)$ pressa, 02 $(7,4 \%)$ excesso de tarefas, $02(7,4 \%)$ falta de atenção do colega, $02(7,4 \%)$ urgência, $02(7,4 \%)$ não uso de EPI, 02 (7,4\%) reencape de agulha, $(3,7 \%)$ quadro reduzido de pessoal e $01(3,7 \%)$ estresse (Tabela 4). Investigação ${ }^{(12)}$ sobre acidentes de trabalho com material perfurocortante em um hospital universitário evidenciou que $17,6 \%$ dos trabalhadores de enfermagem citaram como causa do acidente a fatalidade e a agitação do paciente, 13,7 $\%$ à negligência do colega, $11,8 \%$ à falta de cuidado, $9,8 \%$ pressa e sobrecarga de trabalho e $3,9 \%$ falta de pessoal.

Tabela 4: Distribuição do número e porcentagem dos trabalhadores de enfermagem de um Centro de Terapia Intensiva que sofreram exposição ocupacional a material biológico $(\mathrm{N}=25)$, segundo a causa atribuída à exposição. Sertãozinho, 2007.

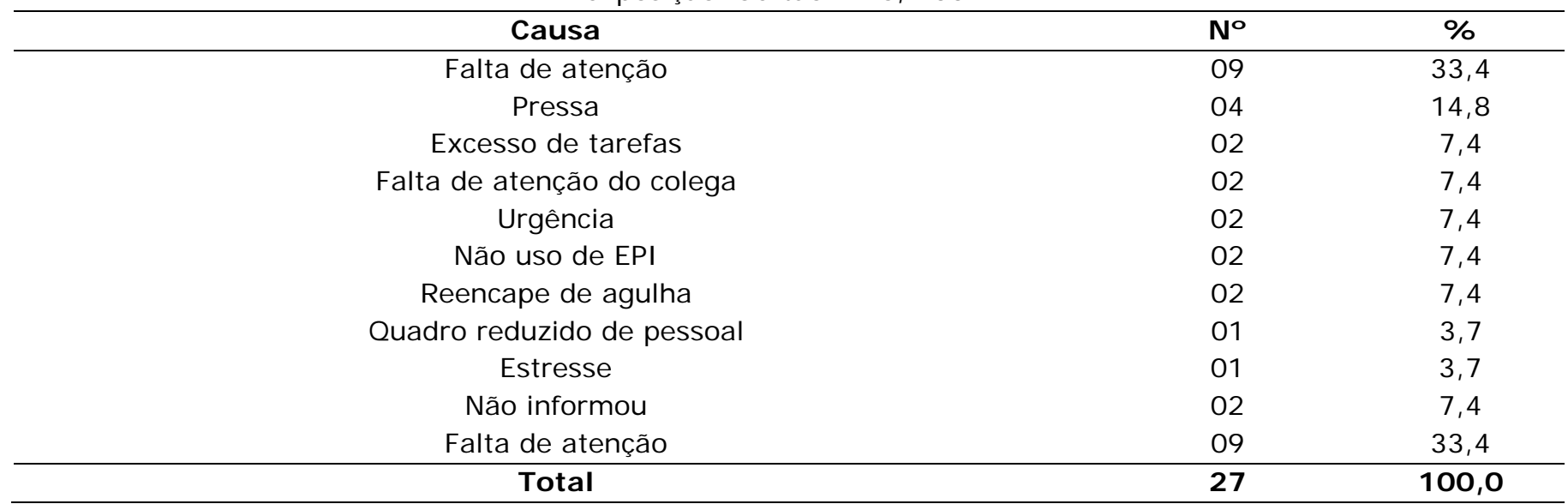

A UTI é uma unidade de cuidado complexo e dinâmico que requer habilidade e rapidez dos profissionais de saúde e também demanda uma elevada carga de trabalho para a equipe de 
enfermagem(19) devido à alocação de pacientes críticos, instáveis, sujeitos à constantes alterações hemodinâmicas e iminente risco de morte, os quais exigem atenção ininterrupta e tomada de decisões imediatas. Assim, torna-se necessário o conhecimento sobre o mecanismo de ocorrência de acidentes envolvendo material biológico e a avaliação do processo de trabalho, para que medidas, capazes de tornar o ambiente mais seguro, sejam propostas.

O uso de EPI é de extrema importância para a prevenção de exposição à material biológico, conferindo maior segurança para profissionais e pacientes. Porém, para que a segurança se torne efetiva é necessário não somente a adesão ao uso, mas também a maneira como são utilizados, limpos e conservados $^{(20)}$. Estes mesmos autores apontam que a formação e preparo dos enfermeiros é fundamental para um exercício profissional seguro, devendo as instituições formadoras investir em educação conscientizadora.

\section{CONI DERAÇÕES FI NAIS}

Este estudo possibilitou a caracterização dos profissionais de enfermagem de um Centro de Terapia Intensiva que sofreram exposição ocupacional a material biológico bem como dos acidentes. Houve predominância das exposições percutâneas e o sangue foi o fluido corporal mais freqüentemente envolvido nos acidentes e apesar de a maioria dos sujeitos relatar ter participado de cursos e ou palestras sobre o uso das precauçõespadrão observou-se que em muitas situações eles não utilizavam os equipamentos de proteção individual.

O conhecimento, em si, não assegura a adoção de comportamentos seguros no trabalho, além de que é necessário avaliar que tipo de conhecimento os treinamentos têm enfocado. Incluir aspectos relacionados à mudança de comportamento, bem como estimular a auto-promoção da saúde podem contribuir para mudar o panorama de acidentes ocupacionais com material biológico entre os trabalhadores da área da saúde.

Assim, torna-se premente a revisão das estratégias utilizadas nos programas de educação permanente de modo que estes sejam capazes de contribuir para a conscientização dos profissionais sobre os riscos ocupacionais e conseqüentemente valorizarem sua segurança durante o exercício profissional.

\section{REFERÊNCI AS}

1. Ippolito G, Puro V, Heptonstall J, Jagger J, De Carli G, Petrosillo N. Occupational human immunodeficiency virus infection in health care workers: worldwide cases through September 1997. Clin Infect Dis. 1999;28(2): 365-83.
2. Do AN, Ciesielski CA, Metler RP, Hammett TA, Li J, Fleming PL. Occupationally acquired human immunodeficiency virus (HIV) infection: national case surveillance data during 20 years of the HIV epidemic in the United States. Infect Control Hosp Epidemiol. 2003; 24(2): 86-96.

3. Rapparini, C. Occupational HIV infection among health care workers exposed to blood and body fluids in Brazil. Am J I nfect Control. 2006; 34(4) :237-40

4. Ministério da Saúde. Secretaria de Atenção à saúde. Departamento de Ações Programáticas estratégicas. Exposição a materiais biológicos. Brasília (Brasil): Ministério da Saúde; 2006. 76 p.

5. Garner JS. Guideline for isolation precautions in hospitals. Infect Control Hosp Epidemiol 1996; 17(1): 53-80.

6. Siegel JD, Rhinehart E, Jackson M, Chiarello L, Helth Care Infection Control Practices Advisory Committee. Guidelines for Isolation Precautions: preventing transmission of infectious agents in healthcare settings. Am J Infect Control. 2007; 35(10): S65-164.

7. Centers for Disease Control and Prevention. Update U. S. Public Health Service Guideline for the management of occupational exposure to HBV, HCV, and HIV and Recommendations for postexposure prophylaxis. MMWR Morb Mortal Wkly Rep. 2001; 50(RR-11): 1-42.

8. Canini SRMS, Gir E, Hayashida M, Machado AA. Acidentes perfurocortantes entre trabalhadores de enfermagem de um hospital universitário do interior paulista. Rev Latino-am Enfermagem. 2002; 10(2): 172-8.

9. Correa CF, Donato M. Biossegurança em uma unidade de terapia intensiva- a percepção da equipe de enfermagem. Esc. Anna Nery. 2007;11(2): 197204.

10. Chan R, Alexander M, Chan E, Chan V, Ho B, Lai $\mathrm{CY}$, et al. Nurses' knowledge of and compliance with universal precautions in an acute care hospital. Int J Nurs Stud. 2002; 39(2): 157-63.

11. Clarke SP, Rockett JL, Sloane DM, Aiken LH. Organizational climate, staffing, and safety equipment as predictors of needlestick injuries and near-misses in hospital nurses. Am J Infect Control. 2002; 30(4): 207-16.

12. Brandi S, Benatti MCC, Alexandre NMC. Ocorrência de acidente do trabalho por material perfurocortante entre trabalhadores de enfermagem de um hospital universitário da cidade de Campinas, Estado de São Paulo. Rev. esc. enferm. USP. 1998; 32(2): 124-33.

13. Campins $M$, Torres $M$, Varela $P$, López Clemente V, Gascó A, de la Prada $M$ et al. Needlestick injuries in health care workers: analysis of non preventable risk factors through standard precautions. Med Clin (Barc) . 2009; 132(7):251-8 
14. Moongtui W, Gauthier DK, Turner JG. Using peer feedback to improve handwashing and glove usage among Thai health care workers. Am J Infect Control. $2000 ; 28(5): 365-9$.

15. Ciorlia LAS, Zanetta DMT. Significado epidemiológico dos acidentes de trabalho com material biológico: hepatites $\mathrm{B}$ e $\mathrm{C}$ em profissionais da saúde. Rev Bras Med Trab. 2004;2(3): 191-9.

16. Nishide VM, Benatti MCC, Alexandre NMC Ocorrência de acidente do trabalho em uma unidade de terapia intensiva. Rev Latino-am Enfermagem. 2004; 12(2): 204-11.

17. Florêncio VB, Rodrigues CA, Pereira MS, Souza ACS. Adesão às precauções padrão entre os profissionais da equipe de resgate do corpo de Bombeiros de Goiás. Rev. Eletr. Enf. [Internet]. 2003 [cited 2009 set 29];5(1)43-8. Available from: http://www.fen.ufg. br/revista/revista5 1/adesao. html.

18. Ministério do Trabalho e Emprego. Portaria $\mathrm{n}$-485, de 11 de novembro de 2005. Aprova a Norma Regulamentadora no 32. Segurança e Saúde no Trabalho em Estabelecimentos de Saúde. Brasília: Ministério do Trabalho e Emprego; 2005.

19. Inoue KC, Matsuda LM. Dimensionamento da equipe de enfermagem da UTI-adulto de um hospital ensino. Rev. Eletr. Enf. [Internet]. 2009 [cited 2009 set 29];11(1):55-63. Available from: http://www.fen.ufg.br/revista/v11/n1/v11n1a07.htm 20. Tipple AFV, Souza ACS, Souza CPS, Paiva EMM, Pereira MS. Equipamentos de Proteção Individual: uso e manuseio por alunos em uma instituição de ensino odontológico. Revista ABO Nacional. 2003; 11(3) : 153-61.

Artigo recebido em 30.08.08.

Aprovado para publicação em 08.09.09.

Artigo publicado em 30.09.09. 